\title{
Distribuição de Médicos e Oftalmologistas no Brasil e Portugal
}

\section{Distribution of Physicians and Ophthalmologists in Brazil and Portugal}

Thiago Gonçalves dos Santos MARTINS $\triangle^{1,2,3}$, Rufino SILVA ${ }^{2}$, Paulo SCHOR ${ }^{1}$

Acta Med Port 2020 Oct;33(10):635-637 - https://doi.org/10.20344/amp.13571

Palavras-chave: Brasil; Médicos; Oftalmologistas; Portugal

Keywords: Brazil; Ophthalmologists; Physicians; Portugal

\section{INTRODUÇÃO}

Portugal e Brasil são países lusófonos com sistemas de saúde universais e situação económica distinta. Em Portugal, a constituição estabelece que todos têm direito à proteção da saúde por meio de um Serviço Nacional de Saúde e que cabe ao Estado garantir uma cobertura eficiente de recursos humanos em saúde. No Brasil, de 1960 até 2015, o número de médicos para cada mil habitantes quadruplicou, e o aumento da oferta de postos de trabalho médico devido à expansão do sistema de saúde contribuiu para esse crescimento. Em 2017, o Brasil apresentava uma taxa de 10,2 médicos recém-formados por 100 mil habitantes, a mesma de Portugal e acima dos Estados Unidos (5,5). ${ }^{1,2}$ O objectivo deste artigo é analisar alguns fatores comparativos sobre a distribuição de médicos e oftalmologistas desses dois países.

\section{Distribuição de médicos e oftalmologistas no Brasil}

O crescimento do número de vagas e de faculdades gerou aumento do número de médicos formados, mas não foi acompanhado por uma melhoria a nível da qualidade de ensino de acordo com os resultados dos exames promovidos anualmente pelo Conselho Regional de Medicina de São Paulo desde 2005. O exame realizado pelos formandos de 30 das faculdades de medicina do estado de São Paulo avaliadas em 2015 demonstrou que, dos 2726 participantes, $48,13 \%$ não acertaram em pelo menos $60 \%$ das 120 questões. Em sete anos de história, este exame também constatou que $46,7 \%$ dos participantes saíram das faculdades mal preparados, sem condições mínimas de exercer Medicina.

A irregularidade na distribuição dos médicos pode ser evidenciada quando determinamos a razão médicos/1000 habitantes em diferentes regiões brasileiras. Os menores valores ocorrem nas regiões Norte $(1,16)$ e Nordeste $(1,41)$, os quais estão abaixo da média nacional de 2,11 médicos por 1000 habitantes. Em contraste, as outras três regiões apresentam médias acima da nacional, como a região Sudeste $(2,81)$.
Em 2018, quando a população do Brasil era de 208494000 habitantes, havia 452801 médicos, o que corresponde a uma razão de 2,17 médicos por mil habitantes. Essa densidade de profissionais é semelhante à de países desenvolvidos como a Inglaterra (2,8 por mil habitantes), Estados Unidos (2,7 por mil habitantes) e Canadá (2,7 por mil habitantes). No Brasil, 55,3\% dos médicos e $42 \%$ da população estão situados na região Sudeste, enquanto que na região Nordeste encontramos $17,4 \%$ dos médicos e $27,8 \%$ do total da população brasileira.

Para se comparar regiões e estados quanto à presença e concentração de médicos especialistas usamos a razão especialista/generalista (médico sem título de especialista). Sendo os generalistas os médicos que se formaram na faculdade de medicina e não realizaram o internato.

Em 2018, quando existiam 280 mil especialistas no Brasil, havia 1,67 especialistas para cada generalista. A diferença entre esta razão nos diversos estados pode parecer pouco acentuada, mas o número absoluto de especialistas difere bastante. A maior parte dos médicos especialistas encontram-se na região Sul $(2,27)$ contra a menor concentração na região Norte $(1,06)$. Embora tenha ocorrido um aumento das faculdades de Medicina no interior do país, essas não conseguiram fixar médicos no local onde estudaram. Há que realçar que $43 \%$ das vagas de Medicina ainda estão nas capitais. ${ }^{2}$ As distribuições irregulares também podem ser explicadas pela irregularidade na distribuição das vagas de internato. No ano de 2019, o Brasil tinha 56 255 vagas de residência médica por ano, concentradas em apenas $5 \%$ dos municípios brasileiros. A distribuição das vagas de internato acompanha as diferenças sociodemográficas do Brasil: a região Norte apresenta o menor número de programas. Essa oferta é desigual dentro das próprias regiões; na região Sul, por exemplo, apenas $5 \%$ dos municípios possuem vagas para formação especifica dos internos. Na região Centro-Oeste são $2 \%$ dos municípios, e nas regiões Nordeste $3 \%$ dos municípios. Essa tendência repete-se na região Sudeste com $7 \%$ dos municípios e

1. Departamento de Oftalmologia. Universidade Federal de São Paulo. São Paulo. Brasil.

2. Departamento de Oftalmologia. Universidade de Coimbra. Coimbra. Portugal.

3. Departamento de Oftalmologia. Ludwig Maximilians Universitat. Munique. Alemanha.

$\square$ Autor correspondente: Thiago Gonçalves dos Santos Martins. thiagogsmartins@yahoo.com.br

Recebido: 10 de fevereiro de 2020 - Aceite: 12 de maio de 2020 | Copyright $\odot$ Ordem dos Médicos 2020 
na região Norte com $4 \%$ dos municípios. Isso demonstra a carência e concentração de centros de formação de especialistas médicos no Brasil. ${ }^{3}$

Os oftalmologistas representam $3,6 \%$ de todos os especialistas no Brasil e a sua distribuição desigual é somente o reflexo da distribuição dos médicos. Essa realidade prejudica o atendimento da população com doenças oftalmológicas. De acordo com o censo realizado pelo Conselho Brasileiro de Oftalmologia, apenas $15 \%$ dos municípios brasileiros contam com oftalmologistas, ou seja, $85 \%$ das localidades - e praticamente um quarto dos 201 milhões de habitantes do país - carecem de assistência oftalmológica. Na região Sudeste vive $42 \%$ da população brasileira e 57\% dos oftalmologistas, um profissional para cada 8601 habitantes. Nas regiões Sul, Centro-Oeste e Nordeste estão, respectivamente, 15\%, 7\% e 19\% desses profissionais. Em contraste, no Norte brasileiro há apenas um oftalmologista para cada 30491 habitantes. Essa relação tem piorado ao longo dos anos, com o aumento da população e a manutenção do número de oftalmologistas na região. Todos os estados do Norte apresentam significativa carência de especialistas, onde trabalham $3,8 \%$ dos oftalmologistas. Catorze municípios concentram $50 \%$ de todos os oftalmologistas brasileiros, sendo 10 capitais, três municípios do interior de São Paulo e um do Rio de Janeiro. Noventa e sete por cento dos oftalmologistas trabalham em cidades com mais de 40 mil habitantes. Analisando os censos realizados nos últimos anos pelo Conselho Brasileiro de Oftalmologia nos anos de 2001 e 2014, houve um expressivo aumento do número de oftalmologistas, mas as regiões Norte e Centro-Oeste continuam a apresentar as maiores carências. A proporção de oftalmologistas no Brasil era em 2001 de 1/17 620 habitantes, e em 2014 apresentou uma relação de 1/11 604 habitantes. ${ }^{4}$

A organização hierarquizada do Sistema Único de Saúde (SUS) dificulta ainda mais o atendimento oftalmológico à população brasileira, pois a oftalmologia está situada longe da porta de entrada do sistema. As políticas desenvolvidas pelo Ministério da Saúde colocam o serviço oftalmológico em níveis secundário e terciário de complexidade.

Outra causa da dificuldade de acesso ao atendimento oftalmológico é o número de médicos que trabalham apenas no sistema privado, o que reduz a assistência a grande parte da população dependente do sistema público de saúde. De acordo com os dados do sistema de saúde, $21,6 \%$ dos médicos trabalham exclusivamente no setor público e $26,9 \%$ só atuam no setor privado. Os demais $51,5 \%$, atuam nas duas esferas, a pública e a privada. A desigualdade de concentração dos médicos a favor do setor privado é grande, quando consideradas as populações cobertas pelo Sistema Único de Saúde ( $75 \%$ da população utiliza exclusivamente o SUS) e pela assistência médica suplementar ( $25 \%$ da população, além do direito ao SUS, têm planos ou seguros de saúde).

A presença de médicos nos centros de saúde e estratégia de saúde da família $(23 \%$ dos que atuam no setor público estão nessas estruturas, enquanto $51 \%$ trabalham em hospitais) pode não ser suficiente para a efetivação dos cuidados de saúde primários como porta de entrada no sistema de saúde. Pior ainda é a situação dos cuidados de saúde secundária e especializada do SUS, no qual atuam menos de $5 \%$ dos médicos. A forte atuação de especialistas em consultórios particulares, em contraste com a baixa presença nos ambulatórios do Sistema Único de Saúde, é um grande obstáculo à ampliação, na rede pública, da oferta de assistência médica especializada. De acordo com uma pesquisa realizada entre estudantes do primeiro ano de Medicina em 2018, essa situação permanece inalterada, já que $50,2 \%$ desejam trabalhar em consultório particular, enquanto apenas $19,4 \%$ que desejam trabalhar no SUS. ${ }^{2}$

\section{Distribuição de médicos e oftalmologistas em Portugal}

Em Portugal, existe uma desigualdade menos acentuada na distribuição de médicos com uma relação médico por 10000 habitantes de 38,7 , superior ao número de 18,9 do Brasil. $^{5}$

Em Portugal existe uma assimetria na distribuição de médicos. Há uma concentração de médicos nas zonas do Porto, Coimbra e Lisboa e défice em regiões como Alentejo, Algarve e Beira Interior. O Algarve, com 1900 habitantes por médico, é a região que apresenta a menor cobertura. ${ }^{6}$ Outro dado a ter em conta é que cerca de metade dos estudantes de Medicina em Portugal são da área da Grande Lisboa, o que poderá acentuar ainda mais as assimetrias regionais na distribuição de médicos. No que se refere ao acesso a cuidados de saúde, $26 \%$ da população já tem um seguro privado de saúde. ${ }^{7}$ Em 2015, quando a população do país era de 10368346 habitantes existiam, segundo uma pesquisa realizada pelo Conselho Internacional de Oftalmologia (ICO), 600 oftalmologistas em Portugal, o que corresponde a 57,9 oftalmologistas por milhão de pessoas, com um número de 36,2 oftalmologistas por milhão de pessoas realizando cirurgias de catarata e um número de 50,7 oftalmologistas por milhão de pessoas realizando refração. Comparando com os dados do Brasil, este país apresentava no mesmo período 14000 oftalmologistas, o que representava 67,4 oftalmologistas por milhão de pessoas, 25,3 oftalmologistas por milhão de pessoas que realizam cirurgias de catarata e 58,9 oftalmologistas por milhão de habitantes que se submetam a refração. ${ }^{8}$ A necessidade de atendimento oftalmológico em idosos tem vindo a aumentar em Portugal, já que o país deverá apresentar em 2050 uma percentagem de idosos superior a 35\%. ${ }^{6}$ Em 1971, Portugal apresentava uma percentagem de pessoas acima de 65 anos de 9,7\%. Em 2018, essa faixa etária representava $21,7 \%$ da população, com um rácio de 157,4 idosos para cada 100 jovens. A percentagem de oftalmologistas em Portugal relativamente a todos os médicos especialistas baixou de 4 em 1990 para 2,8\% em 2018, apesar do aumento do número absoluto de 446 oftalmologistas em 1990 para 1096 em 2018. ${ }^{9}$ Quanto à a distribuição territorial de oftalmologistas em Portugal, apesar do aumento do número absoluto de profissionais em todas regiões entre os anos de 2001 e 2018, a área metropolitana de Lisboa continua 
Tabela 1 - Dados obtidos pelo Conselho Federal de Medicina (2018), Conselho Brasileiro de Oftalmologia (2014) e Ordem dos Médicos $(2018)^{2,4,9}$

\begin{tabular}{lcc}
\hline & Brasil & Portugal \\
\hline Médicos/ mil habitantes & 2,18 & 5,21 \\
Oftalmologista (\% de especialistas) & $3,6 \%$ & $2,8 \%$ \\
Região com maior \% de oftalmologistas & Sudeste $(57 \%)$ & Lisboa (44,3\%) \\
Região com menor \% de oftalmologistas & Norte $(3,8 \%)$ & Açores (1\%) e ilha da Madeira (1\%) \\
Gasto com saúde & $3,8 \%$ do PIB & $9,0 \%$ do PIB \\
\hline
\end{tabular}

a concentrar o maior número de especialistas $(44,3 \%)$ e a região do Algarve a menor concentração $(3,1 \%)$, na região continental. Nos Açores e na Ilha da Madeira encontram-se apenas $1 \%$ dos especialistas em oftalmologia. ${ }^{9}$

\section{Comentários finais}

Os dados discutidos neste artigo referem-se aos médicos inscritos no Conselho Federal de Medicina no Brasil e na Ordem dos Médicos em Portugal. Apesar de alguns números Brasileiros serem superiores aos Portugueses, isso acaba por não ter impacto no atendimento da população. Isto deve-se a problemas de mobilidade urbana e isolamento desses especialistas face à população necessitada, o que resulta na concentração de especialistas e em diferenças geográficas e organizacionais a nível dos sistemas de saúde. A comparação da realidade nos dois

\section{REFERÊNCIAS}

1. Oliveira N, Alaves L. Ensino médico, SUS e início da profissão: como se sente quem está se formando? Rev Brase Educ Med. 2011;35:26-36.

2. Scheffer M, Biancarelli A, Cassenote A. Demografia médica no Brasil 2018. São Paulo: Conselho Federal de Medicina; 2018.

3. Campos V, Fidelis F, Silva P, Teixiera A, Batista A. Recorte demográfico da residência médica brasileira em 2019. Revista Consensus. 2019;32.

4. Conselho Brasileiro de Oftalmologia. Censo oftalmológico 2014 [Internet]. São Paulo (SP): CBO; 2014. [consultado 2015 out 25]. Disponível em https://www.cbo.net.br/novo/publicacoes/CensoCBO2014.pdf.

5. Organização Mundial de Saúde. Estatísticas Sanitária Mundial. Genebra: OMS; 2012. países reforça a ideia de que um simples aumento no número de especialistas não é o suficiente para melhorar a qualidade do atendimento oftalmológico à população. Além disso, Portugal possui um maior investimento em saúde, que passou de $8,4 \%$ do PIB em 2000 para 9,0\% em 2018, enquanto o Brasil tem um gasto atual de $3,8 \%$ do PIB em Saúde (Tabela 1). Essas diferenças em termos de investimentos também pesam na qualidade de atendimento da população. $^{9}$

\section{CONFLITOS DE INTERESSE}

Os autores declaram não haver conflitos de interesses.

\section{FONTES DE FINANCIAMENTO}

Não houve suporte financeiro para este trabalho.

6. Correia I, Veiga P. Geographic distribution of physicians in Portugal. Eur J Health Econ. 2010;11:383-93.

7. Campos L. Uma perspetiva sobre o Relatório da OCDE "Health at a Glance 2017". Acta Med Port. 2018;31:9 -11.

8. Resnikoff S, Lansingh V, Washburn L, Felch W, Gauthier T, Taylor H, et al. Estimated number of ophthalmologists worldwide (International Council of Ophthalmology update): will we meet the needs? $\mathrm{Br} \mathrm{J}$ Ophthalmol. 2020;104:588-92.

9. Fundação Francisco Manuel dos Santos. (2013). Retrato de Portugal: indicadores 2011. Paris: PORDATA. [consultado 2020 jan 30] Disponível em https://www.pordata.pt/Portugal. 